\title{
Title: Digital marketing: a strategic and holistic approach
}

\section{[Interview with Sarah Quinton]}

Many people believe that they are continually bombarded by marketing messages. Yet when they are not on the receiving end, they may be engaging in their own marketing activities. This has become even more pronounced in the digital era, creating the need to step back and see how organizations have tried to shape and adapt to digital marketing, and how scholars have studied and written about this challenging subject.

A constructive place to look is the 2016 International Journal of Management Reviews article "The Digital Journey: Reflected Learnings and Emerging Challenges," by Sarah Quinton, of the Oxford Brookes Business School, Oxford Brookes University; and Lyndon Simkin, now of Henley BusinessSchool, University of Reading; bothinthe United Kingdom.

The authors point to what they call the "digitalization of marketing," an ever-changing, complex, and elaborate journey taken by companies and other organizations in the era of computers, smartphones, tablets, and similar devices. "Digital marketing," they write, "involves computer-mediated environments that allow consumers, firms and other stakeholders to create, interact and access digital content."

Quinton says that key findings in the article that nonspecialists in the field should be aware of include, in her words:

- Digitalization involves adopting a strategic mind- set and orientation that embraces how digital technologies might optimize all aspects of a business. Digital marketing is one aspect of this.

- Digital marketing is dynamic and shaped by both firms and consumers.

- The digital marketing journey has begun and will continue to increase in momentum. It has changedtheworld of consumptionand will continue to do so.

- Digitalization of marketing is not the sole property of large firms. It is within the grasp of SMEs [small to mediumsized enterprises] across all sectors, including B2B [business-to-business].

- All organizations should inhabit the spaces their consumers exist in, and in the twenty-first century thisinvolves participating in a digital journey.

Some of the difficulties of providing successful and effective digital marketing may be attributed to such factors as its relatively recent and ever-changing nature, the need for both traditional online and mobile engagement, specialized technical skills along with content-related skills, and the need for some to run expensive and resource-consuming parallel operations with print and perhaps traditional media such as radio and television. After all, some organizations may market purely in the digital realm, but many others must combine more conventional methods when undertaking their digital journeys.

"Integration of online marketing and mobile is possible," Quinton says,

but challenging as organizations face legacy IT systems, possibly unskilled or unsurestaffand often a lack of clarity over their objectives for both digital and mobile marketing. The choice of platforms and tools should always be relevant to the audience and what is trying to be achieved.

Trying to engage across every possible platform as they constantly proliferate is a fruitless exercise.

The digital era has brought highly specific reporting and measuring tools. However, just because a particular piece of marketing gets a lot of hits or interaction may or may not mean that it is truly successful and effective. Digital marketing that receives relatively less attention can still be valuable, although in different ways. 


\section{According to Quinton:}

There are some marketing academics and practitioners who argue that we are using outdated approaches to try to measure the evolving brave new world of technologically enabled marketing. To some extent I agree as digital requires a new mind-set and goes beyond just implementing a new set of tools. If we narrowourselvesto using onlytheestablished measures and gauges weare likely to miss real insight. The relevance of what is measured and how must relate to the purpose of the digital marketing. Success may be customerco-creation of NPD [new product development], and quantity of activity may not relate to quality

of activity. A lot of interaction may be less influential than a few key influencers' referrals, for example. Value can be generated beyond sales, such as value of reputation enhancement, through customer service recovery via social media. Value in terms of authenticity of interactions with consumers/followersrather than constant trigger marketing offering discounts.

One segment that has become prominent in this realm is content marketing, in which companies and other organizations create articles, graphics, audio, or video with their own content that can make for somewhat blurry lines between journalism and company- sponsored messages. Quinton posits that

content marketing is of lesser importance than contextually relevant marketing. However well written and engaging the content may be, if the context is not right for the consumer they will not engage. Ethically, marketers and journalists have a duty to make explicit to consumers the nature of theircommunication, throughclearly labelled and badged material. Journalists and publications run the risk of losing credibilityand trust if they do not do so and blur the lines for their readers.

The article by Quinton and Simkin provides a big- picture view of a vital and competitive subject. Digital marketing requires practitioners to think and act in novel ways. "The emerging digital culture," the authors write, "and its reshaping of consumer experience requires a new and different mind-set incorporating a more holistic and strategic perspective that encourages integration." 